Nouveaux cahiers de la recherche en éducation

Groux, D. et Baillot, A. (2007). Langue, littérature, culture à l'épreuve de l'autre. Paris : L'Harmattan

\title{
Annabelle Caron
}

Volume 12, numéro 2, 2009

URI : https://id.erudit.org/iderudit/1017471ar

DOI : https://doi.org/10.7202/1017471ar

Aller au sommaire du numéro

Éditeur(s)

Faculté d'éducation, Université de Sherbrooke

ISSN

1911-8805 (numérique)

Découvrir la revue

Citer ce compte rendu

Caron, A. (2009). Compte rendu de [Groux, D. et Baillot, A. (2007). Langue, littérature, culture à l'épreuve de l'autre. Paris : L'Harmattan]. Nouveaux cahiers de la recherche en éducation, 12(2), 245-247.

https://doi.org/10.7202/1017471ar d'utilisation que vous pouvez consulter en ligne.

https://apropos.erudit.org/fr/usagers/politique-dutilisation/ 
de littérature jeunesse francophone. Puisque la liste comporte 10 pages, il aurait été pertinent de regrouper le tout par thèmes afin que le lecteur puisse avoir davantage de points de repère.

Quoi qu'il en soit Lecteurs engagés, cerveaux branchés est un ouvrage qui plaira certainement aux intervenants du milieu scolaire, car il s'agit d'un complément pertinent à un programme de littératie. En effet, cette approche pédagogique novatrice s'avère tout à fait utile pour enseigner explicitement les stratégies de compréhension en lecture, car elle favorise le modelage. L'enseignant est donc à la fois un modèle et un guide. L'élève est quant à lui un être actif et réflexif.

Isabelle Carignan

Université de Sherbrooke

Groux, D. et Baillot, A. (2007). Langue, littérature, culture à l'épreuve de l'autre. Paris: L’Harmattan.

\section{Présentation}

À la question tentant de définir ce qu'est l'éducation comparée, les auteurs qui ont participé au recueil intitulé Langue, littérature, culture à l'épreuve de l'autre diversifient leurs réponses en démontrant qu'il s'agit d'apprendre des autres, échanger, analyser, supputer, rivaliser d'imagination, comparer ce qui se fait ailleurs, établir un lien, confronter, rapprocher ou faire un parallèle.

Cet ouvrage parait dans le cadre d'une revue bisannuelle qui propose des pistes de recherche en France et ailleurs dans le monde au sujet de l'éducation comparée. Elle permet d'approfondir la recherche comparée sous un éclairage multiple qui favorise le dialogue entre différents champs de pratique en sciences sociales et en sciences humaines. Elle s'adresse autant aux praticiens qu'aux chercheurs. Pour ce faire, elle utilise, entre autres, des arguments de nature épistémologique et institutionnelle. Ce premier numéro suggère des pistes de comparaison afin de se «remettre en question, ou remettre sur l'ouvrage, son rapport à l'autre» (p. 9). L'introduction écrite par Baillot annonce rapidement les couleurs de la première publication de cette collection. Comparer ou se comparer est un acte délibéré qui ne peut que soulever les différences et les particularités. Cette forme de confrontation devient un geste qui soulève ce qui nous définit en propre et ce qui nous distingue.

Pour sa part, Groux propose une définition de l'éducation comparée inspirée de Raymond Aron:

L'éducation comparée est l'étude scientifique de l'éducatif en tant que tel. Elle fait appel à la comparaison (historique, géographique) pour mieux analyser et comprendre ses objets d'étude. Elle implique la thématisation de l'éducatif à l'aide de concepts, par définition, suffisamment généraux pour être transversaux (p. 49).

Quant à l'épistémologie en éducation comparée, il s'agit de «l'étude des conditions de possibilité qui permettent la pertinence d'une éducation comparée, c'est-à-dire qui justifient la validité d'une comparaison, soit a priori, soit a posteriori: l'éducation comparée est alors prise dans un 
sens plein» (p. 17). La leçon à propos de l'éducation comparée de Louis Porcher nous fait découvrir un nombre important de variables qu'il faut considérer lorsqu'il s'agit de procéder à une comparaison. Cela demande un effort supplémentaire pour tenir compte de facteurs qui seraient sinon évacués ou ignorés.

Or, comme Groux le mentionne, l'éducation comparée peine à se hisser au rang de «discipline reconnue» (p. 33). Dans nombre de pays, on préfère utiliser les termes d'interculturalité, philosophie éducative, études internationales, etc. Toutefois, il y a un intérêt renouvelé pour cet espace de recherche à qui on attribue la paternité à Marc-Antoine Jullien, de Paris. Dès 1817, il associait le développement de la science de l'éducation en lien avec les autres nations afin de s'étendre et de se perfectionner comme le font les autres sciences.

Plusieurs types d'éducation sont signalés, soit l'éducation comparée interne, l'éducation comparée externe et l'éducation comparée verticale. Un exemple d'éducation comparée interne se traduirait par étudier des élèves de différents niveaux socioéconomiques tout en gardant en tête les valeurs transmises par un système d'éducation qui se veut le même peu importe la situation économique ou le lieu géographique des apprenants. L'égalité et l'équité étant des concepts importants dans ces formes d'analyse, voici comment ils pourraient être définis : "L'égalité étant l'égalité d'objectifs, il faut qu'ils acquièrent la culture par hypothèse, et l'équité étant la différence des moyens, les moyens adaptés à ce que sont les élèves» (p. 17). Dans un tel contexte, il s'agit de comprendre l'éducation dans toute sa complexité et ne pas omettre des variables nécessaires à la compréhension du phénomène.

En contrepartie, une éducation comparée externe, en reprenant l'exemple des élèves, met plutôt en parallèle le parcours d'apprenants qui évoluent dans des pays différents. Il s'agit alors d'évaluer des paramètres qui seront semblables en considérant des systèmes scolaires différents, des âges et des niveaux de scolarité différents selon les nations, etc.

Finalement, l'éducation comparée verticale a pour objet l'étude de l'éducation comparée dans le temps et l'espace. En effet, d'un point de vue historique, plusieurs comparaisons démontrent qu'il ne s'agit pas d'une préoccupation récente, mais bien d'une question qui titille des chercheurs depuis l'époque de la Grèce antique.

\section{Une application pratique: de la didactique des langues à la linguistique}

Plusieurs approches existent en éducation comparée. La didactique comparée est la dernière née. Selon Jean-Pierre Cuq, «les scientifiques qui se réclament de la didactique comparée incarnent cette différence» (p. 68). Par ailleurs, la didactique comparée peut être enfin l'occasion de se confronter avec de grandes disciplines de référence comme la linguistique, la psychologie ou les sciences de l'éducation.

Plusieurs auteurs dans cet ouvrage rappellent que pour effectuer une comparaison, il faut utiliser des éléments comparables. Les contextes, les unités, les liens peuvent être très différents. Cette condition doit être respectée pour faire émerger une comparaison valable. Si Weber, Durkheim ou Mauss ont déjà évoqué la validité comme étant une condition essentielle aux comparaisons, l'objectivation 
est aussi un préalable nécessaire. En effet, il ne s'agit pas seulement de faire abstraction de ses propres croyances, préjugés ou a priori personnels comme le suggère Claude Lévi-Strauss (1958), mais bien de «développer une méthode d'observation qui permet non seulement une objectivité personnelle, mais qui pourra être acquise par tous les observateurs» (p. 41).

Conséquemment, l'éducation comparée favorise l'avancement de la recherche. Toutefois, il appert qu'elle peine encore à s'imposer en tant que discipline. Plusieurs pistes épistémologiques sont abordées dans cet ouvrage. Toutefois, même lorsqu'il s'agissait d'un article qui traitait de cet aspect, les auteurs en viennent à la conclusion que ce sont les assises institutionnelles qui sont les plus chambranlantes. En effet, c'est surtout d'un problème de reconnaissance dont souffre l'éducation comparée.

En conclusion, des réalisations intéressantes ont été accomplies. La publication d'un manuel d'histoire commun aux Allemands et aux Français intitulé Histoire/Geschichte L'Europe et le monde depuis 1945 en est un bel exemple. Un entretien avec Étienne François raconte la genèse de ce projet qui prit forme en 2003 à l'occasion du quarantième anniversaire du traité de l'Élysée. Pour aller au-delà des préjugés et des images toutes faites qui nous empêchent de voir la réalité des autres, il faut oser. L'éducation n'est pas si différente des autres disciplines, scruter un problème sous divers angles pour en saisir l'essence peut faire émerger de nouvelles pistes de solution.

Annabelle Caron

Université de Sherbrooke

Dolz, J. et Simard, C. (2009). Pratiques d'enseignement grammatical. Points de vue de l'enseignant et de l'élève. Québec: Les Presses de l'Université Laval.

\section{Présentation}

L'ouvrage qui comprend 11 articles de chercheurs de pays francophones, Belgique, France, Suisse et Québec, focalise sur les pratiques d'enseignement-apprentissage de la grammaire en salle de classe. Ce collectif permet des réponses à de nombreux questionnements tantôt sur les interventions des enseignants tantôt sur les manières dont les élèves-apprenants s'approprient des contenus grammaticaux. Cette première publication de la collection «Recherches en didactique du français », de l'Association internationale pour la recherche en didactique du français, témoigne d'un intérêt novateur porté en didactique du français, entre autres, par ses apports méthodologiques de recherche. Sans conteste, il met en lumière ce qui caractérise les diverses démarches didactiques dans la réalité de la classe et leur répercussion chez les élèves.

En introduction, Dolz et Simard présentent les changements qu'a connus l'histoire de la grammaire scolaire française, en décrivant succinctement les approches traditionnelle, communicative et renouvelée de la grammaire. Résistances ou espoirs démesurés, l'implantation de la grammaire nouvelle fut, selon leurs observations, inégalement appliquée en salle de classe. Mieux connaitre les pratiques effectives en classe contribuerait, selon eux, à une réelle transposition didactique. 